\title{
Schönheit und Schutz der Natur an der Atlantikküste bei Praia do Forte, Bahia (Brasilien)
}

\author{
Hilke Steinecke, Heidrun Janka, Roland Rudolph \& Karl-Heinz Schulmeyer
}

\begin{abstract}
Natural habitats are endangered in various regions of Brazil. On the other hand there is a number of successful conservation projects. The well-known TAMAR-project has been established in order to protect sea turtles. In the vicinities of Praia do Forte, situated approximately $80 \mathrm{~km}$ in the north of Salvador/Bahia, there is a nature reserve of Atlantic rainforest. In this article the local habitat conservation activities are summarized.

Zusammenfassung

Viele Naturräume in Brasilien sind stark bedroht. Es gibt aber auch sehr erfolgreiche Naturschutz-Projekte. Das vermutlich bekannteste ist das TAMAR-Projekt, das sich dem Schutz der Meeresschildkröten widmet. Um den Ort Praia do Forte, etwa 80 km nördlich von Salvador (Bahia), gibt es außer den geschützten Schildkröten-Stränden ein Naturreservat mit atlantischem Regenwald. Die Naturschutzaktivitäten in diesen Gebieten werden vorgestellt.
\end{abstract}

\section{Das Exkursionsgebiet}

Praia do Forte, ein ehemaliges Fischerdorf, liegt direkt am Atlantik, etwa $80 \mathrm{~km}$ nördlich von Salvador da Bahia (12 $55^{\prime}$ Süd, $38^{\circ} 29^{\prime}$ West) im Bezirk Mâta de Sao Joao. Es hat sich in den letzten Jahren zu einem beschaulichen touristischen Ort mit guter Infrastruktur entwickelt. Hier gibt es zahlreiche Unterkünfte, Restaurants und Kunsthandwerksläden. Von Hochhäusern und anderen großen Hotelbauten ist Praia do Forte glücklicherweise verschont geblieben. Um Grundstücksspekulationen zu vermeiden und den dörflichen Charakter des Ortes zu erhalten, dürfen Fremde hier keine Häuser kaufen. Auf den z.T. noch unbefestigten Lehmstraßen herrscht ein munteres Treiben, ohne dass man Angst vor Kriminalität haben muss, wie es in der Millionenstadt Salvador der Fall ist. Man kann deshalb dort relativ sorglos den $12 \mathrm{~km}$ langen Strand entlang wandern und dabei verschiedene Tiere und Pflanzen beobachten.

Direkt hinter dem weißen Sandstrand schließt sich ein dichter Gürtel aus Kokospalmen an, weshalb die Küste auch Costa dos Coqueiros genannt wird. Dahinter befinden sich ausgedehnte Weißdünen-Gebiete mit gleißend hellem Sand. Die Erkundung der dort wachsenden Pflanzen (insbesondere Kakteen, Orchideen, Bromelien) ist sehr lohnend. An wenigen Stellen der Küste sind noch Reste von Mangrove-Vegetation und des atlantischen Regenwaldes (Mâta Atlântica) erhalten.
Naturschutz und Ökotourismus dominieren in Praia do Forte. Zu dieser Entwicklung hat beigetragen, dass 1972 ein deutscher Industrieller den Ort mitsamt der etwas außerhalb gelegenen mittelalterlichen Ruine (Casa de Torre de Garcia d'Avila) und dessen weitläufige Umgebung (mehrere Tausend Hektar) aufgekauft hat. Die Zerstörung der Landschaft wurde verboten und für das Gebiet sollte ein verträglicher, ökologisch orientierter Tourismus aufgebaut werden. Das Vorhaben ist gelungen. Heute können geführte Wanderungen in die Schutzgebiete, Jeep-Ausflüge oder Walbeobachtungs-Touren gebucht werden. Internationale Anerkennung genießt das TAMAR-Projekt zum Schutz der Meeresschildkröten. Es handelt sich um ein sehr erfolgreiches brasilianisches Naturschutzprojekt. Weltweit sind Meeresschildkröten bedroht. In verschiedenen Ländern gibt es deshalb Schutzprojekte.

\section{Das TAMAR-Projekt}

An der Küste Brasiliens kommen fünf der sechs Meeresschildkröten-Arten vor, nämlich Echte und Unechte Karettschildkröte (Eretmochelys imbricata und Caretta caretta), Bastard- (Lepidochelys olivacea), Suppen- (Chelonia mydas) und Lederschildkröte (Dermochelys coriacea). Die Tiere können beachtliche Größen entwickeln. Eine ausgewachsene Karettschildkröte hat einen $1 \mathrm{~m}$ langen Panzer und wird $150 \mathrm{~kg}$ schwer, die Suppenschildkröte erreicht ein Maximalgewicht von $350 \mathrm{~kg}$ und eine Panzerlänge von $120 \mathrm{~cm}$. 


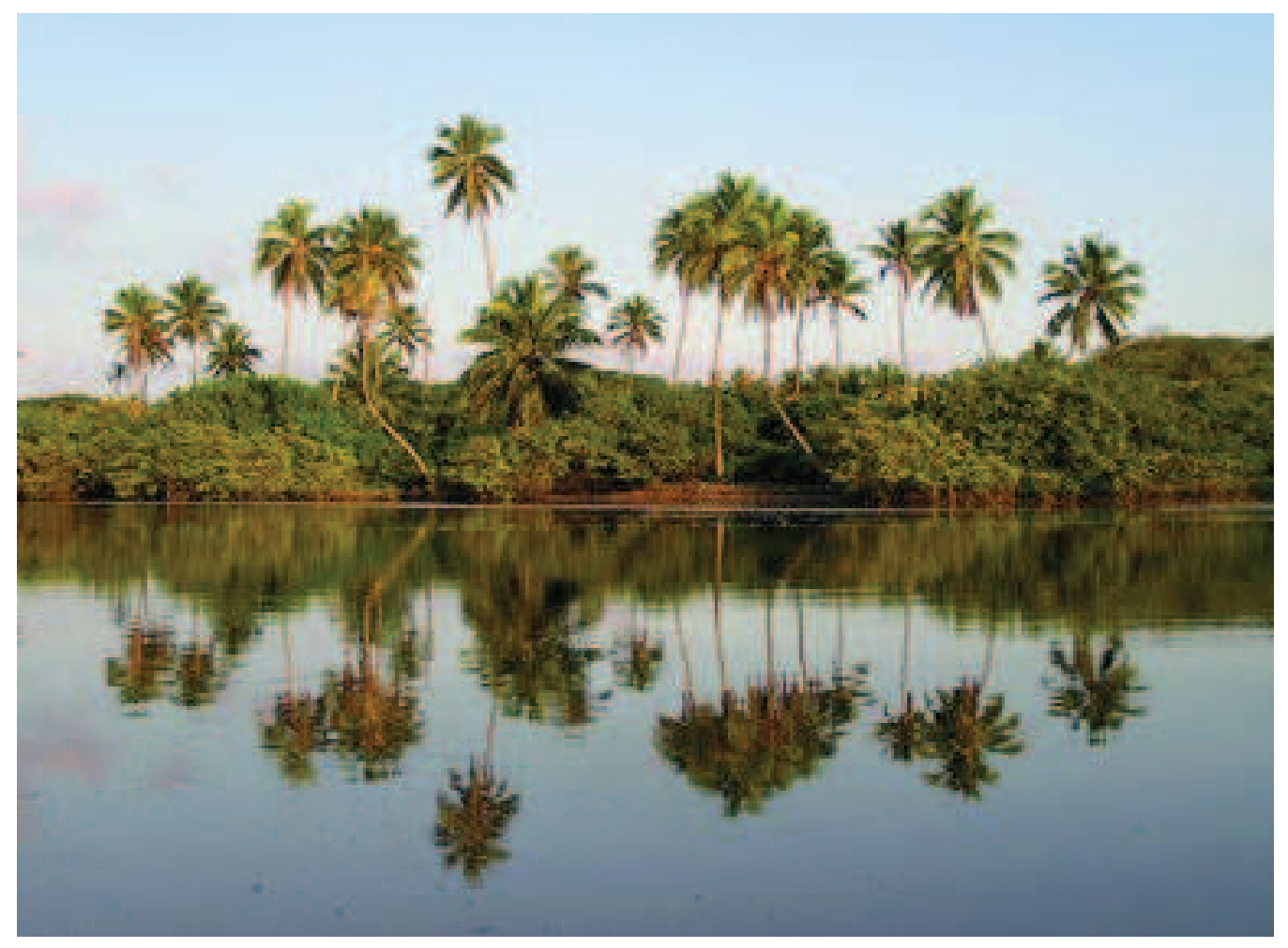

Mit einem Gewicht von $65 \mathrm{~kg}$ und einem $60 \mathrm{~cm}$ langen Panzer gehört die Bastard-Schildkröte zu den kleineren Vertretern.

Die Echte Karettschildkröte hat den auffälligsten und schönsten Panzer, weshalb sie früher häufig gejagt wurde und extrem gefährdet ist. Jungtiere kommen in Gewässern an der Nordost-Küste Brasiliens vor, Eier werden aber nur an Stränden der Nordhälfte Bahias abgelegt. Nester der Unechten Karettschildkröte sind dagegen zwischen Rio de Janeiro und Seripe (kleinster Bundesstaat Brasiliens, nördlich von Bahia gelegen) anzutreffen. Typisch für diese Art ist der kräftige Kiefer, der den Tieren beim Zerbrechen ihrer Hauptnahrung (Mollusken) sehr nützlich ist. Nur ganz selten taucht an Brasiliens Stränden die Lederschildkröte auf. In den letzten Jahren wurden nur 7 Weibchen bei der Eiablage an der Küste von Espírito Santo beobachtet.

Abb. 1: Kleine Lagune mit Kokospalmen bei Praia do Forte.
Die Schildkröten-Bestände gingen Mitte des 20. Jh. rapide zurück und es war zu befürchten, dass die Meeresschildkröten in absehbarer Zeit ganz verschwunden sein würden. Ihre Populationen verringerten sich schnell durch das $\mathrm{Ab}$ schlachten ausgewachsener Tiere für den Verzehr (Suppenschildkröte) und zur Gewinnung von Schildpatt (Karettschildkröte), aufgrund von Eierraub durch Menschen und Tiere sowie durch das Verfangen von Schildkröten in Fischernetzen. Eine große Gefahr geht auch von strandnaher künstlicher Beleuchtung aus, weil dadurch die frisch geschlüpften Schildkröten, die sich normalerweise nach dem Mondlicht richten, die Orientierung verlieren und nicht zum Wasser finden. Es gibt Beobachtungen, dass frisch geschlüpfte Schildkröten direkt in ein am Strand brennendes Feuer gelaufen und verbrannt sind. Um einen weiteren Rückgang der Meeresschildkröten zu verhindern, wurde 1979 vom Brasilianischen Umweltinstitut IBAMA das TAMAR-Projekt gegründet. Der Name leitet sich vom portugiesischen Tartaruga Maritima 

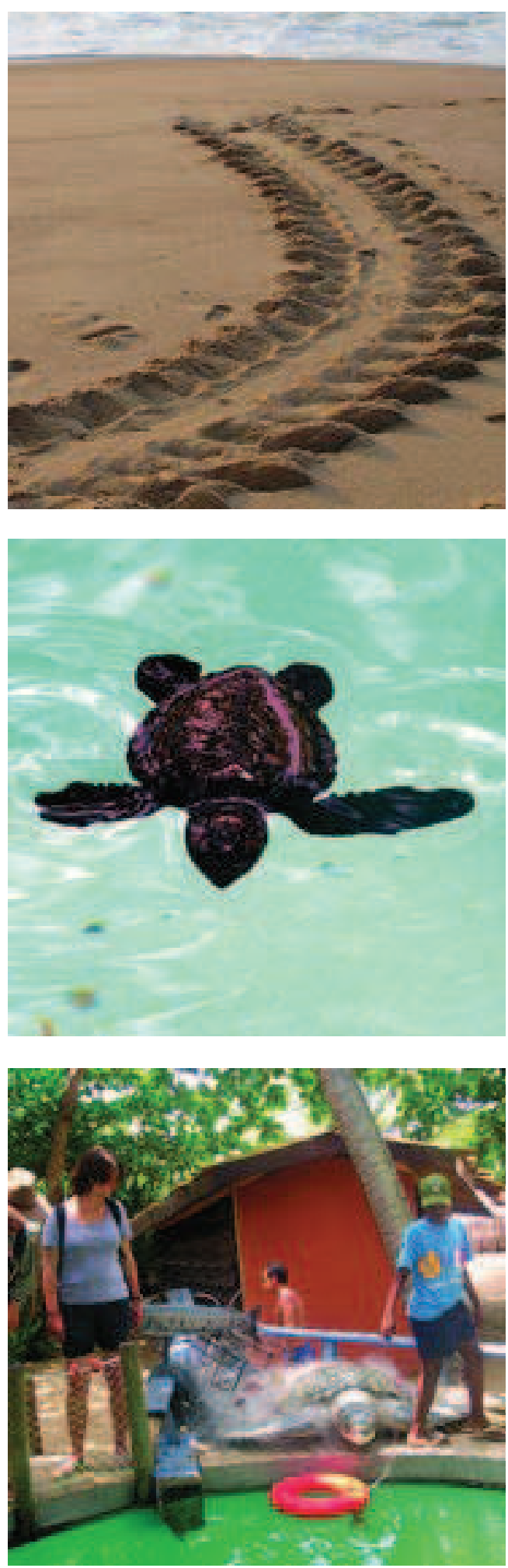

(= Meeresschildkröte) ab. Das Projekt wird von der größten Erdölgesellschaft Brasiliens sowie von verschiedenen Naturschutz-Organisationen unterstützt, darunter auch dem WWF Deutschland.

1980 wurde zunächst eine umfassende Bestandsaufnahme der Schildkröten-Vorkommen gemacht, so dass anschließend gezielte Schutzmaßnahmen durchgeführt werden konnten. Im Laufe der Jahre wurden bis heute 22 Kontrollund Schutz-Stationen in acht Bundesstaaten errichtet. An allen Stationen erforschen Wissenschaftler und Studenten Biologie, Ökologie und Bestände der Meeresschildkröten. Manche Stationen werden kontinuierlich betreut, die anderen sind nur in der Zeit zwischen Eiablage und Schlüpfen der Jungtiere besetzt. SchildkrötenSaison ist von September bis März: die HauptEiablagezeit reicht von November bis Januar, die meisten Jungtiere schlüpfen im Februar und März. Die Weibchen kommen zur Eiablage an ihren Geburtsstrand zurück. Die einzelnen Individuen legen aber nicht jedes Jahr ihre Eier ab, sondern kommen z. T. nur alle drei Jahre zur Eiablage an Land. Die Eier werden durch die Sonnenwärme ausgebrütet.

Da um Praia do Forte besonders viele Meeresschildkröten vorkommen und ihre Eier im Sand vergraben, wurde hier das Nationale Zentrum zum Schutz und zur Beobachtung von Meeresschildkröten errichtet. Es wurden ideale Bedingungen für die Schildkröten geschaffen. So wurden auch Lichtquellen in Strandnähe „schildkrötenfreundlich" umgerüstet. Eier, die zu nah am Wasser abgelegt sind, werden eingesammelt und an kontrollierten Strandabschnitten wieder vergraben. Die Gelege werden z. T. durch Gitteraufsätze und mehrmalige nächtliche Kontrollen geschützt. Das Freilassen der frisch geschlüpften Schildkröten wird überwacht, so dass Verluste durch natürliche Feinde an Land nahezu ausgeschlossen werden. Die örtliche Bevölkerung ist

Abb. 2 (oben): Spuren einer Meeresschildkröte.

Abb. 3 (Mitte): Junge Schildkröte im Schaubecken. Abb. 4 (unten): Führung durch die TAMAR-Station. 
in den Schildkröten-Schutz einbezogen: aus ehemaligen Fischern, Schildkröten-Fängern und Eierräubern sind Schildkröten-Schützer geworden. Insgesamt sind rund 1200 Personen am Schildkröten-Schutz beteiligt. Das Ergebnis lässt sich sehen. Vor 20 Jahren schlüpften an Brasiliens Küsten pro Jahr nur noch 20000 Schildkröten, heute sind es wieder 600000 . Mittlerweile ist unter der Obhut des TAMAR-Projektes die fünfmillionste Meeresschildkröte geschlüpft und die ersten Jungtiere sind bereits geschlechtsreif. Allein in Praia do Forte erblicken aktuell jährlich nach 45-60 Tagen Brutzeit 22000 Schildkröten-Babies das Licht der Welt. Ranger patroullieren am Strand und verwischen schon kurz nach Sonnenaufgang jegliche Spuren der Schildkröten.

Es wird sehr viel Wert auf Umweltbildung gelegt. Im Besucherzentrum gibt es Schaubecken mit ausgewachsenen und ganz jungen Schildkröten sowie vielen anderen Meeresbewohnern. Schautafeln und Modelle geben Informationen zur Biologie und Ökologie der Schildkröten sowie zur Arbeit des TAMAR-Projektes. Bereits die Kinder des Ortes identifizieren sich mit dem Schildkröten-Schutz. Bucht man eine Führung durch das Besucherzentrum, erhält man mitunter von ca. 10-jährigen Schulkindern durchaus qualifizierte Erläuterungen. TAMAR betreibt zudem einen „grünen“ Kindergarten mit Vorschule.

Zur finanziellen Unterstützung können Schildkröten-Patenschaften erworben werden. Außerdem werden kulturelle Events wie beispielsweise der Turtle Day organisiert. Im Verkaufsraum kann man sich mit T-Shirts und Kappen mit Schildkrötenemblemen sowie mit Schildkröten-Schmuck, -Tassen und -Spielzeug eindecken. Die Erlöse aus dem Verkauf finanzieren mittlerweile einen großen Teil der laufenden Kosten des Projektes. TAMAR betreibt zwei

Abb. 5 (oben): Blick in die Dünenlandschaft.

Abb. 6 (Mitte): Blühender und fruchtender Melocactus.

Abb. 7 (unten): Bromelien und Kakteen in den Dünen, im Hintergrund der Kokosgürtel.
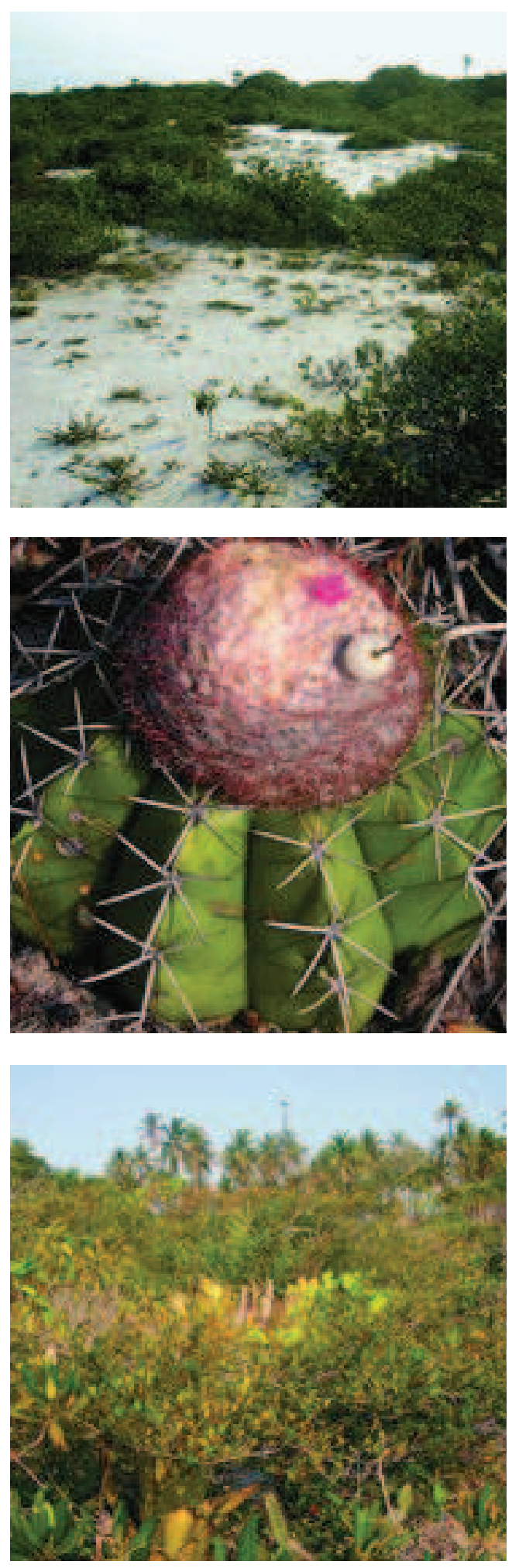

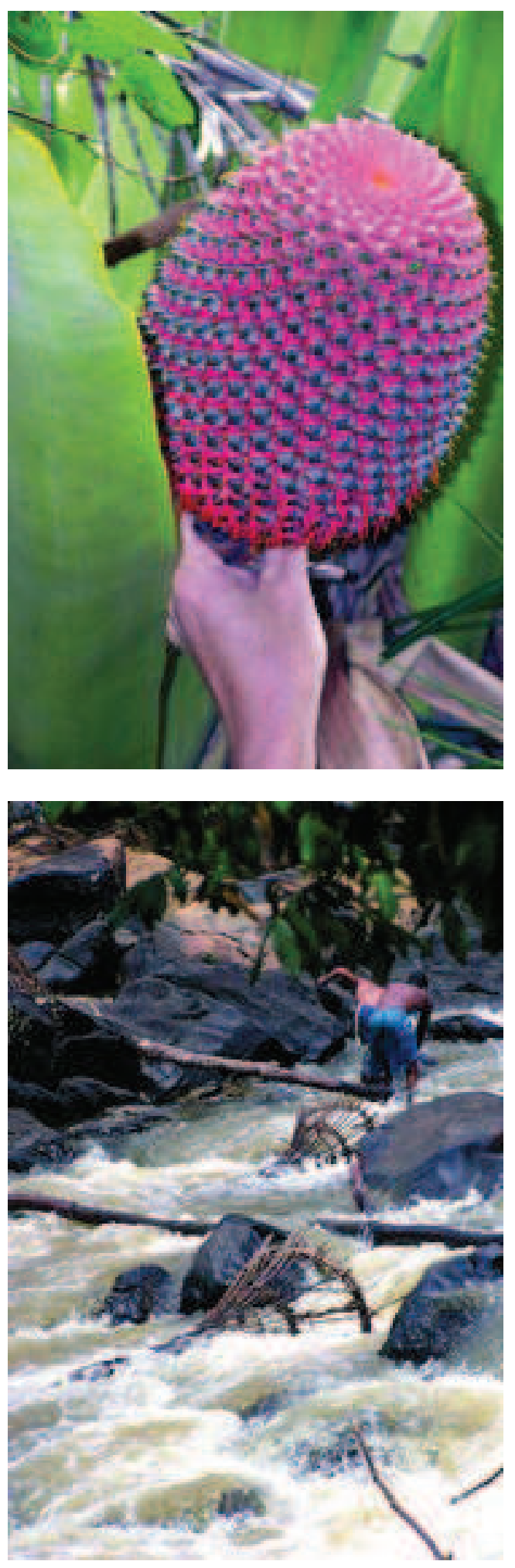

T-Shirt-Manufakturen, wodurch als weiterer positiver Effekt 700 Arbeitsplätze geschaffen wurden, meist in strukturschwachen Regionen.

Das TAMAR-Projekt macht weltweit auf sich aufmerksam. Im Frühjahr 1998 wurde es in einer Ausstellung im Bundesumweltministerium in Bonn vorgestellt. Zahlreiche Reiseveranstalter, die Touren an Brasiliens Küsten organisieren, planen auch den Besuch einer TAMAR-Station ein. Es bleibt zu hoffen, dass das TAMAR-Projekt weiterhin einen so effektiven Meeresschildkröten-Schutz betreibt. Eine nicht abzuschätzende globale Gefahr geht allerdings von der Verschmutzung der Ozeane aus. Außerdem beeinflusst die Umgebungstemperatur während der Brutzeit das Geschlecht der Schildkröten. Weibchen benötigen höhere Temperaturen als Männchen. Naturschützer befürchten, dass es im Zuge der Klimaerwärmung zu einer ungleichen Geschlechterverteilung zu Gunsten der Weibchen kommen kann.

\section{Weißdünen}

Hinter den Schildkrötenstränden und dem ca. $100 \mathrm{~m}$ breiten Kokospalmengürtel schließt sich eine etwa einen halben Kilometer breite Zone mit Weißdünen an. Die Pflanzendecke ist hier stellenweise sehr lückenhaft und es gibt größere Flächen mit strahlend weißem Sand. Das Wasser versickert an solchen Standorten sehr schnell, so dass sich hier nur besondere Spezialisten ansiedeln wie z. B. terrestrische Orchideen mit wasserspeichernden Bulben und Bromelien mit groBen Blattzisternen, in denen sich Regenwasser ansammelt. Die hier vorkommende Aechmea pectinata wächst sowohl terrestrisch als auch epiphytisch bis in Höhenlagen um 1100 m. Zwischen einzelnen Kokospalmen gedeihen auch Cashew-Pflanzen und stachelig beblätterte Nachtschatten-Arten. Unscheinbar sind die hier mitunter nur wenige Zentimeter hohen Vertreter der Eriocaulaceae (z. B. Syngonanthus, Paepalanthus). Ihre winzigen weißen Blüten sind zu

Abb. 8 (oben): Junger Fruchtstand von Aechmea multiflora. Abb. 9 (unten): Fangen von Flusskrebsen. 
kugeligen Köpfchen vereinigt. Der tagsüber großen Hitze sowie der Trockenheit trotzen verschiedene Kakteen. Als besondere Attraktion gibt es in den Dünen Melocactus violaceus. Der Gattungsname leitet sich von lat. melo = Melone $\mathrm{ab}$ und bezieht sich auf die kugelige Gestalt. Melokakteen haben auffällige Rippen und sind stark bedornt. Zum Schutz vor zu großer Hitze sind die Kakteen z. T. in den Sand versenkt, so dass sie leicht übersehen werden. In den Dünen gibt es viele Jungpflanzen mit Sprossdurchmessern von nur ca. $1 \mathrm{~cm}$, aber auch ältere mit etwa $10 \mathrm{~cm}$ dicken kugeligen Sprossen. Mehrere Jahre alte Melokakteen entwickeln an ihrem Scheitel ein borstig-haariges Cephalium. An diesem bilden sich die relativ kleinen, violett gefärbten Blüten. Nach eigenen Beobachtungen werden diese gern von Ameisen aufgesucht.

\section{Atlantischer Regenwald}

An der Ostküste Südamerikas (vor allem im Bereich von Brasilien, Argentinien und Paraguay) gab es zwischen dem 5. und dem 28. Breitengrad (Süd) einst ausgedehnte Regenwälder, die sich über eine Länge von $5000 \mathrm{~km}$ erstreckten. Ursprünglich bedeckte der Atlantische Regenwald eine Fläche von $1 \mathrm{Mio} . \mathrm{km}^{2}$. Heute sind es nur noch $50000 \mathrm{~km}^{2}$. In Bahia ist sein Anteil von $31 \%$ der Landfläche auf $7 \%$ geschrumpft, die verbliebenen Reste sind stark gefährdet.

Bereits die Portugiesen rodeten große Anteile, um Nutzhölzer zu entnehmen. Seit den 1970er Jahren beschleunigte sich die Zerstörung, um Anbauflächen für Soja zu gewinnen. Um die letzten Reste zu retten, wurde 1993 die Mâta Atlântica in 14 Bundesstaaten Brasiliens zum UNESCO-Biosphärenreservat erklärt.

Einen Eindruck von der Artenvielfalt in einem (sekundären) Atlantischen Regenwald kann man in einem geschützten Waldrest, nur $2 \mathrm{~km}$ außerhalb von Praia do Forte, gewinnen. Das Schutzgebiet Reserva de Sapiranga umfasst eine Fläche von 600 ha, ist eingezäunt und darf nur im Rahmen geführter Touren betreten werden.

Abb. 10 (oben): Ficus mit Luftwurzeln.

Abb. 11 (unten): Schmetterlings-Ansammlung.
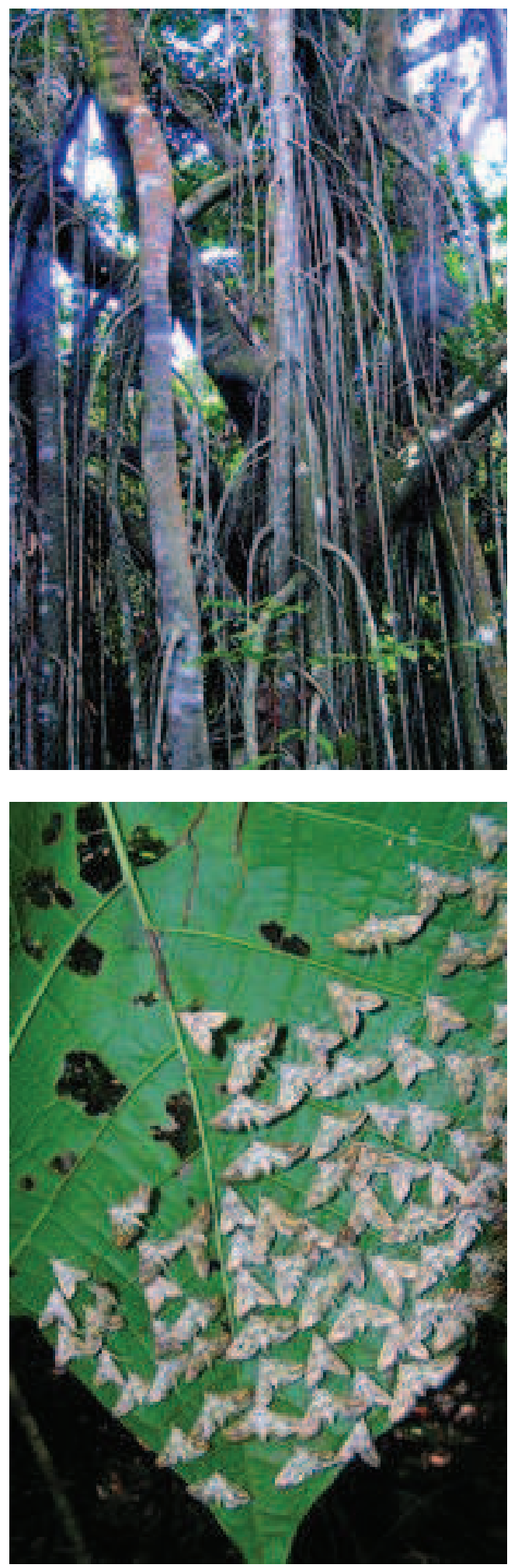


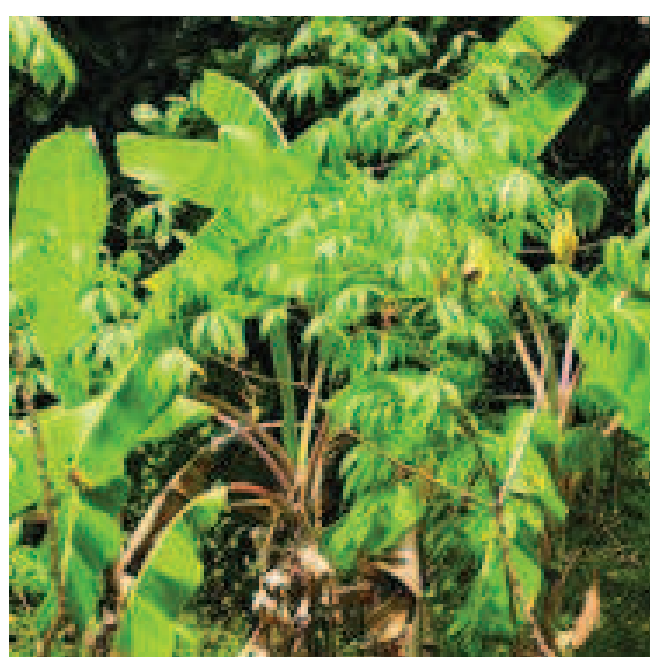

Die Wanderungen dauern zwischen einer halben und fünf Stunden. Urwaldriesen mit ausladenden Brettwurzeln gibt es hier nur wenige. Einzelne große Ficus-Bäume fallen durch ihre Luftwurzeln auf. Eine weitere charakteristische Baum-Art dieses Gebietes ist die Leguminose Bowdichia virgilioides, die ein wertvolles Bauholz liefert. Im Wald sind außerdem verschiedene Palmen anzutreffen. An lichten Standorten gibt es viele Lianen und Epiphyten, darunter auch Vanilla chamissonis. Besonders beeindruckend ist Aechmea multiflora mit überkopfgroßen Blütenständen, die aufgrund ihrer roten Hochblätter eine gute Fernwirkung haben. Wir trafen ein stattliches, blühendes Exemplar an, das epiphytisch auf einer Palme wuchs. Die Pflanze bildete eine $2 \mathrm{~m}$ breite Blattrosette mit ebenso langen Blättern aus. Der Durchmesser des Blütenstandes betrug 15-20 cm.

Im Schutzgebiet können zudem viele Tiere beobachtet werden, darunter zahlreiche Insekten: Über die Wege laufen Blattschneiderameisen. Vorsicht ist vor Treiberameisen geboten. Eciton ist eine neuweltliche Gattung dieser Ameisen. Sie bauen kein festes Nest wie die meisten Ameisen, sondern rotten sich zu einem lebendigen Nest, das Biwak genannt wird, zusammen. Die Nester werden an Baumstämmen oder in Erdhöhlen angelegt. Auf Beutezug gehen die Ameisen in Schwärmen oder Gruppen. Wenige Stiche dieser Ameisen reichen aus, um bei empfindlichen Personen heftige allergische Reaktio- nen auszulösen. Ein besonders markanter Bewohner des Gebietes ist das Weißbüscheläffchen (Callithrix jacchus). Ursprünglich kam es nur an der Nordküste Brasiliens vor, wurde aber auch im Süden des Landes eingeführt. In ihrem ursprünglichen Verbreitungsgebiet sind die Weißbüscheläffchen stark bedroht, jedoch vermehren sie sich in ihrer neuen Heimat in Südost-Brasilien sehr gut und werden dort auf Plantagen mitunter sogar zu einer Plage. In Erdhöhlen lebt das Langnasen-Gürteltier (Dasypus septemcunctus). Nur selten zu sehen bekommt der Besucher vermutlich den Borstenbaumstachler (Chaetomys subspinosus). Es handelt sich um einen Baumbewohner, der sich nur langsam im Geäst fortbewegt. Tagsüber schläft er in Baumhöhlen oder Erdlöchern, um in der Nacht auf Nahrungssuche zu gehen. Er frisst vor allem Früchte und Nüsse. Während der Führung gelangt man auch zum Fluss Rio Pojuce. Hier wird demonstriert, wie nach alter Tradition mit Hilfe von Reusen Flusskrebse gefangen werden.

Einen Überblick über die Tier- und Pflanzenwelt und den Schutz des Gebietes erhält der Besucher in einem kleinen Informationszentrum am Rande des Waldes, wo auch einige typische Tiere des Gebietes wie Papageien und Schlangen gehalten werden.

\section{Dank}

Der Besuch der Küstenregion um Praia do Forte fand im Rahmen der Reise in die Chapada Diamantina (wir berichteten darüber) im September 2005 statt. Für die finanzielle Unterstützung aus Mitteln des GERDA-Hopf-Reisestipendiums danken wir der Gesellschaft „Freunde des Palmengartens".

\footnotetext{
Internetseiten

http://www.tamar.org/br/ingles/default.htm www.br-online.de/bayern2/radiowissen/schildkröte-meerbeitrag-ID1220522118182.xml

www.greenpeace-magazin.de/index.php?id=3389 www.unterwasserwelt.de/html/meeresschildkröte brasiliane. html
}

Abb. 12: Kleinbäuerliche Pflanzung mit Bananen und Maniok am Rande des Regenwaldes. 